\section{AB1130 RELIABILITY OF ULTRASOUND MEASUREMENT OF HYALINE CARTILAGE THICKNESS IN RHEUAMTOID ARTHRITIS}

Edoardo Cipolletta, Emilio Filippucci, Andrea DI Matteo, Marco DI Carlo, Walter Grassi. Polytechnic University of Marche, Rheumatology Unit, "Carlo Urbani" Hospital, Jesi, Italy

Background: Only few studies investigated the role of ultrasound (US) in the assessment of hyaline cartilage in rheumatoid arthritis (RA).

Recently, a positive correlation was found between the US measurement of the metacarpal head cartilage thickness (MCT) and both the anatomical MCT and the radiographic joint space width ${ }^{1}$.

Objectives: To evaluate inter- and intra-observer reliability in the assess ment of MCT in RA patients and healthy subjects; to compare the agreement of the sonographers in the assessment of the MCT using different methods (i.e. semiquantitative and quantitative); to determine the interobserver smallest detectable difference (SDD) of MCT measured by US.

Methods: US assessment was performed by two rheumatologists on 160 metacarpophalangeal (MCP) joints of 10 healthy subjects and 10 patients with RA (according to 2010 ACR/EULAR classification criteria) using a MyLab Twice (Esaote Biomedica, Genoa, Italy) equipped with a linear very high frequency probe (i.e. $10-22 \mathrm{MHz}$ )

To assess inter-observer reliability, the hyaline cartilage of metacarpal head from II to $\mathrm{V}$ digits of both hands were examined independently on the same day by two rheumatologists (an experienced musculoskeletal sonographer and an investigator with limited US training).

To assess intra-observer reliability, all the subjects were re-scanned using the same scanning protocol and the same US setting by one sonographer after a week.

Hands were scanned with the MCP joints in maximal flexion (approximately $90^{\circ}$ ). The hyaline cartilage of all the metacarpal heads was scanned in longitudinal and transverse views in the central portion of the metacarpal head. Particular attention was paid on maintaining the probe in a position providing an angle of $90^{\circ}$ between the direction of the US beam and the cartilage surface ${ }^{2}$.

MCT was scored both semi-quantitatively (using a five-grade scoring system $^{3}$ ) and quantitatively (using the average value of the longitudinal and transverse measures)

The inter- and intra-observer agreements for assessing the MCT with the semiquantitative scoring system were calculated using Cohen's kappa and interpreted according to Landis and Koch.

The inter- and intra-observer agreements for assessing the MCT with the quantitative scoring system were calculated using intraclass correlation coefficients (ICC) and their 95\% confident intervals $(95 \% \mathrm{CI})$.

The SDD was determined using Bland-Altman $95 \%$ limits of agreement method.

Results: The inter- and intra-observer agreements for the semiquantitative assessment of the MCT were moderate $[\mathrm{k}=0.59(95 \% \mathrm{Cl} \quad 0.35-0.83)$ and $\mathrm{k}=0.63(95 \% \mathrm{Cl} 0.39-0.87)$, respectively].

Considering all the measurements, a substantial inter-observer $[\mathrm{ICC}=0.88$ $(95 \% \mathrm{Cl} \quad 0.82-0.92)]$ and intra-observer $[\mathrm{ICC}=0.88 \quad(95 \% \mathrm{Cl} \quad 0.87-0.94)]$ agreements for the quantitative assessment of MCT were found.

The SDD of the MCT measurement was: $0.11 \mathrm{~mm}$ for both longitudinal and transverse scans and $0.09 \mathrm{~mm}$ for the average of the two measures.

Conclusion: This study provides evidence in favor of the reliability of semiquantitative and quantitative US methods for assessing MCT in RA. Further studies are required to determine standard reference values of MCT by US in healthy subjects.

\section{REFERENCES}

[1] Mandl P, Supp G, Baksa G, et al. Relationship between radiographic joint space narrowing, sonographic cartilage thickness and anatomy in rheumatoid arthritis and control joints. Ann Rheum Dis. 2015;74:2022:7.

[2] Torp-Pedersen S, Bartels EM, Wilhjelm J, Bliddal H. Articular cartilage thickness measured with US is not as easy as it appears: a systematic review of measurement techniques and image interpretation. Ultraschall Med. 2011;32:54-61

[3] Filippucci E, da Luz KR, Di Geso L, et al. Interobserver reliability of ultrasonography in the assessment of cartilage damage in rheumatoid arthritis. Ann Rheum Dis. 2010;69:1845-8.

Disclosure of Interests: None declared

DOI: 10.1136/annrheumdis-2019-eular.250
Edoardo Cipolletta, Emilio Filippucci, Andrea DI Matteo, Marco DI Carlo,

Fausto Salaffi, Walter Grassi. Polytechnic University of Marche, Rheumatology Department, "Carlo Urbani" Hospital, Jesi, Italy

Background: Conventional radiography is the standard imaging modality to detect joint damage in rheumatoid arthritis (RA). Ultrasound (US) allows for a direct visualization of hyaline cartilage. To date, only few studies investigated the role of US in the assessment of cartilage damage in RA.

Objectives: To compare US qualitative and quantitative assessments of cartilage thinning at metacarpal head $(\mathrm{MH})$ in $\mathrm{RA}$ patients and in an age-, sex- and height-matched healthy controls $(H)$. To correlate cartilage damage and clinical parameters in RA.

Methods: US examination was performed on 318 metacarpophalangea (MCP) joints of 40 consecutive RA patients and on $320 \mathrm{MCP}$ joints of 40 age-, height- and sex-matched $\mathrm{H}$ using a MyLab Twice (Esaote Biomedica, Genoa, Italy), equipped with a high frequency linear probe (up to $22 \mathrm{MHz}$.

RA patients were enrolled according to the 2010 RA classification criteria The hyaline cartilage of $\mathrm{MH}$ from II to $\mathrm{V}$ digits of both hands was examined with the MCP joints in maximal flexion.

Each $\mathrm{MH}$ was scanned in longitudinal and transverse views. Particular attention was paid on maintaining the probe in a position providing an angle of $90^{\circ}$ between the direction of the US beam and the cartilage surface ${ }^{1}$.

Cartilage thickness (CT) was assessed both semi-quantitatively (using a reliable qualitative five-grade scoring system ${ }^{2}$ ) and quantitatively (using the mean value of longitudinal and transverse measurements of the CT) Finally, association between cartilage damage and clinical parameters was assessed.

Results:

Semiquantitative score: Cartilage thinning (grade 2, 3 and 4) was found in at least one $\mathrm{MH}$ in $23 \mathrm{RA}$ patients $(57.5 \%)$ and in $4 \mathrm{H}(10.0 \%)$ $(p<0.01)$. A significantly higher prevalence of cartilage damage (grade 2 3 and 4) at joint level was found in RA patients (86 MCP joints, $27.0 \%$ ) in comparison with $\mathrm{H}(13 \mathrm{MCP}$ joints, 4.1\%) $(\mathrm{p}<0.01)$.

Quantitative assessment: CT of the $\mathrm{MH}$ ranged from 0.0 to $1.10 \mathrm{~mm}$ $(0.60 \pm 0.26 \mathrm{~mm}$, mean $\pm S D)$ in $R A$ patients and from 0.41 to $1.08 \mathrm{~mm}$ $(0.67 \pm 0.12 \mathrm{~mm}$, mean $\pm S D)$ in $H$. Male had a thicker hyaline cartilage than female, both in RA patients $(p<0.01)$ and in $H(p<0.01)$. No significant difference was found between left and right side, both for RA patients $(p=0.48)$ and healthy subjects $(p=0.94)$.

Detailed quantitative measurements of $\mathrm{CT}$ of $\mathrm{MH}$ are reported in table 2.

Abstract AB1131 Table 2. US cartilage thickness measurement for each digit in RA patients and healthy controls.

\begin{tabular}{lcccc}
\hline & $\begin{array}{c}\text { RA patients } \\
(\mathrm{n}=39)\end{array}$ & $\begin{array}{c}\text { Healthy subjects } \\
(\mathrm{n}=40)\end{array}$ & $\begin{array}{c}\text { Mean difference, 95\% Cl } \\
(\mathrm{mm})\end{array}$ & $\begin{array}{c}\mathrm{P} \\
\text { value }\end{array}$ \\
\cline { 2 - 3 } & Mean $(\mathrm{mm})$ & Mean $(\mathrm{mm})$ & & \\
\hline II MH & 0.62 & 0.75 & $0.13 ; 0.06-0.21$ & $<0.01$ \\
III MH & 0.57 & 0.64 & $0.07 ; 0.02-0.13$ & $\mathbf{0 . 0 1}$ \\
IV & 0.61 & 0.64 & $0.03 ;-0.03-0.09$ & 0.35 \\
MH & & & & \\
V MH & 0.62 & 0.65 & $0.03 ;-0.02-0.09$ & 0.20 \\
Sum & 2.43 & 2.68 & $0.25 ; 0.03-0.46$ & $\mathbf{0 . 0 2}$ \\
\hline
\end{tabular}

95\%Cl: 95\% confidence interval; L: left; $\mathbf{M H}$ : metacarpal head; R: right; RA: rheu matoid arthritis; SD: standard deviation; US: ultrasound.

A significant association was found between the CT value and age ( $r=$ $0.528, p<0.001)$, disease duration $(r=-0.376, p=0.005)$ and grade of the semiquantitative scoring system $(r=-0.80, p<0.001)$. No association was found between CT and BMI, weight, ACPA positivity and RF positivity. Conclusion: This study demonstrated that a significantly higher prevalence of cartilage damage was found in RA patients using both the semiquantitative score and the quantitative assessment. In particular, in RA patients the hyaline cartilage of $\mathrm{II}$ and $\mathrm{III} \mathrm{MH}$ is thinner in comparison with $\mathrm{H}$. Finally, a significant association was found between the CT values and disease duration and age.

\section{REFERENCES}

[1] Torp-Pedersen S, Bartels EM, Wilhjelm J, Bliddal H. Articular cartilage thickness measured with US is not as easy as it appears: a systematic review of measurement techniques and image interpretation. Ultraschall Med. 2011;32:54-61 